\title{
Towards Underlying Radio Technologies for LTE Based Public Safety Networks
}

\author{
Guopeng Zhang ${ }^{1}$, Ao $\mathrm{Li}^{2}$, Yong Zhou' ${ }^{*}$, Yao $\mathrm{Du}^{2}$ \\ ${ }^{1}$ School of Computer Science and Technology, China University of Mining and Technology, Xuzhou, China, \\ 221116. \\ ${ }^{2}$ School of Information and Electrical Engineering, China University of Mining and Technology, Xuzhou, \\ China, 221116.
}

* Corresponding author. Tel: +86 516 83591707; email: yzhou@cumt.edu.cn

Manuscript submitted September 23, 2015; accepted December 27, 2015.

doi: 10.17706/jcp.12.2.97-104

\begin{abstract}
Public Safety Networks (PSNs) have been recognized as the key to a successful response to emergency and disaster situations. Recently, the 3GPP-LTE cellular networks open possibilities to enhance public safety services. To position the current LTE to meet the requirements of the evolution of PSNs, this paper proposes an approach to investigates the two key radio technologies, i.e., device-to-device (D2D) communications and user equipment (UE) initiated multicast communications. First, due to physical damage to base stations (BSs) or network congestion, wireless infrastructures may not available to users in post-disaster areas. In such a situation, we propose a D2D communication approach which allows two far apart users to communicate directly without the use of BSs. Then we consider that a UE instead of a BS acts as the multicast transmitter. To satisfy the QoS requirements of emergency group communications, an effective opportunistic multicast scheduling approach is proposed at the MAC layer which can minimize the delay of a multicast. To further improve multicast throughput, D2D relaying technique is also integrated into the proposed multicast scheduling scheme, which can combat the multicast bottleneck when one or very few receivers are with very poor channel conditions.
\end{abstract}

Key words: Emergency communications, LTE cellular networks, post-disaster network reconstruction, device-to-device communications, wireless multicast communications.

\section{Introduction}

In recent years, natural disasters (such as the Great East Japan Earthquake and Tsunami) and public safety emergences (such as the U. S. 9/11 Events and the London Subway Bombings) not only result in considerable damage to the affected area and loss of life, but also have profoundly endangered public security. In order to reduce damage and avoid loss of life, it is essential that emergency information could be shared among population within affected area, local public safety agencies and government/public service organizations [1]. It therefore requires that information and communication technologies (ICTs) to be fundamentally changed. For instance, after impact of earthquake, no available communication infrastructure remained due to physical damage to cellular base stations (BSs). Although disaster victims have mobile user equipments (UEs) such as mobile phones and tablets, those UEs become practically useless because there is no BS to support communications and insufficient available power. Similarly, once a public safety incident occurs, a large number of calls might be initiated concurrently and emergency calls within the affected area toward the outside are most probably blocked. The lesson learned from these 
emergent situations is that UEs should have essential functionalities to transmit emergency information (e.g. text, voice, photo, etc.) toward the outside, even with best effort service and even without acknowledgment [2].

Public Safety Networks (PSNs) have been recognized as the key to a successful response to emergency and disaster situations, which allow emergency messages to be sent from disconnected areas and information sharing among disaster victims and evacuation centers. The evolutions of PSNs have followed two major distinct paths: Project 25 (P25) [3] and Terrestrial Trunked Radio (TETRA) [4]. P25 is pursued under the auspices of the U.S. Telecommunications Industry Association (TIA) and TETRA under the European Telecommunications Standards Institute (ETSI). However, both TETRA and P25 intended for the provision of voice and low-speed (about $9.6 \mathrm{~kb} / \mathrm{s}$ ) data services originally [5], [6]. When attempting to explore broadband technologies and provide higher-speed services (with rates of close to $80 \mathrm{~kb} / \mathrm{s}$ in a 50 $\mathrm{kHz}$ wide channel), both TETRA and P25 face the same obstacle as lack of available wireless spectrum resource [7].

Recent years have seen the growing wireless multimedia capabilities offered to consumers by new 3G technologies. The 3G Partnership Project (3GPP) Long Term Evaluation (LTE) mobile broadband services open possibilities to enhance public safety services daily and special field operations [8]. Yet, adding the broadband technique to existing PSNs is not feasible as P25 and TETRA are essentially the narrowband solutions offering mainly voice services. It is feasible to combine the existing narrowband P25\TETRA control core infrastructure (with its sufficient voice quality and a customized feature set) with the real time broadband capabilities of a commercial LTE network. The main benefit in the use of LTE for PSNs involves having a large-scale deployment of LTE networks and a large ecosystem worldwide, which allow less expensive equipment based on unified standards. These standards can be adopted by all PSNs globally and share scale with non-public-safety applications of LTE. To enable LTE to become a competitive broadband ICT for PSNs, the 3GPP is currently targeting the availability of the two key communication schemes expected by PSNs, i.e., device-to-device (D2D) communication [9] and group/multicast communication [10] in LTE Release 12. However, in order to position the current LTE to meet the requirements of the evolution of PSNs, the following main research challenges arise which motivate the proposed work.

1) When part of a network fails, emergency actions order that the remainder of the network must continue to provide services to the greatest extent possible. In such a situation, D2D communications enable two or more UEs to communicate directly without the use of network infrastructure, and can also offload heavy cellular traffic onto D2D radio links [11]. Although 3GPP has been working on defining and developing D2D related services and functionalities (what is generally called the proximity services (ProSe) in 3GPP [12]) over past years, most of current studies only focus on single-hop route/link between two D2D UEs. As most of UEs are out of range of network cells after disasters, it is necessary that forthcoming LTE-based PSNs (LTE-PSNs) support multi-hop communications between any two far apart D2D UEs [13]. The related issues, such as route discovery, power control, interference management and protocol implementation should be well addressed in the context of LTE-PSNs.

2) Individuals of different emergency first-responders are organized as different groups based on types of skills or activities to be performed, e.g. individuals in a fire brigade or a police department. A first-responder need to coordinate the individuals' actions, causing their communications to be treated as group/multicast communications. Multicast to group members is not confined to speech. Multimedia data, e.g. text, image, video and so on can also be sent in parallel to speech [8]. Hence, PSNs must provide Quality of Service (QoS) controls to manage multicast service for diverse traffics. However, current LTE standards support QoS controls only for unicast communications - reach only a single UE [2]. Besides, in contrast to traditional cellular multicast service, it may be UEs rather than 
BSs in PSNs that perform multicast since most of UEs are out of BS coverage. This introduces additional challenges due to the limited capability of UEs.

3) Besides no available infrastructures, the great challenges in post-disaster situations also include lack of energy necessary for UEs to operate. To cope with the battery power shortage, new ICTs are required to improve energy efficiency for current LTE UEs.

This paper aims to apply advanced radio technologies in LTE-based PSNs. More specifically, we shall focus on the areas of PSN oriented D2D multi-hop communications, wireless group/multicast communications and energy-saving radio technologies. These topics are prioritised by the numerous UK and global public safety communities, councils and companies, such as ETSI, TIA, National Public Safety Telecommunications Council (NPSTC), TETRA \& Critical Communications Association (TCCA) [14], AT\&T, Qualcomm, Alcatel Lucent, Deutsche Telekom, Ericsson, Huawei, Rogers Wireless and Vodafone, etc.

\section{Research Hypothesis and Objectives}

As LTE is expected to become the most widely deployed broadband communication system, the key research hypothesis of our approach is that LTE techniques can be exploited in conjunction with multi-hop D2D communication technique and wireless group/multicast communication technique to meet the special requirements (e.g., QoS and energy efficiency) of PSNs and provide necessary high-quality emergency services.

\subsection{Novelty}

To the best of our knowledge, until now no such work has been done to bring the two key technologies together towards an ecological LTE-based PSN design. Most of the previous efforts only address some partial issues of this topic. More specifically:

1) It has not been carefully studied yet how to set feasible transmit powers for multi-hop D2D transmitters that keeps the interference to cellular UEs within the allowed interference level. Additionally, there is no design on the network information to realize multi-hop route discovery between two far apart D2D UEs, and there is no related method to establish a multi-hop route between two D2D UEs.

2) There are no discussions on the efficient group/multicast communication schemes for emergency services (e.g. push-to-talk (PTT) services) in current 3GPP framework, especially when BSs are damaged and thus UEs rather than BSs perform the multicast. Correspondingly, a dedicated end-to-end QoS framework to prioritize multicast services and perform access and congestion control is also needed within the LTE-based PSN ecology.

3) There are no discussions on how to reduce UE energy consumption and on how to extend network lifetime in emergency communication scenarios. Besides, to meet the special QoS requirements of multimedia unticast (single-hop or multi-hop) and multicast communications, physical layer (PHY) techniques and media access control (MAC) layer techniques should be jointly considered from the perspective of cross-layer design.

Ecological LTE-based PSN is still in its infancy. The proposed paper intends to provide timely solutions towards LTE-based PSN by jointly considering the above techniques in an innovative way. The novelty of our approach is significant because it is increasingly being recognized that effective communications are key to a successful response to emergency and disaster situations. Moreover, our approach proposed here may lay the foundations to large-scale commercialization of LTE-based PSNs in the future.

\subsection{Objectives}

The overall aim of the proposed approach is to develop novel radio technologies to improve the emergency communication service in LTE-based PSNs. The specific goal includes (1) multi-hop D2D route 
discovery algorithm and power and interference control strategies, (2) optimized multicast scheduling scheme over D2D and cellular links, and (3) cross-layer design based QoS framework for energy efficient unicast/multicast communications in LTE-based PSNs. The measurable objectives are:

1) To develop intelligent power control strategies for multi-hop D2D communications that allows D2D transmitters to opportunistic access the spectrum actively in use by cellular communications.

2) To design dedicated route discovery packets as well as distributed dynamic spectrum access protocol that can establish single-hop or multi-hop route between two D2D UEs.

3) To develop energy efficient multicast scheduling strategies over emergency D2D and cellular radio links, and to evaluate and compare the solutions (in terms of QoS and energy efficiency performances and implementation complexities) as well as to assess their suitability for PSN environments;

4) To formulate the cross-layer optimization problems that correlate the multi-layer dynamics of emergency unicast/multicast with realistic conditions, e.g., imperfect channel states estimates, packet outage and heterogeneous QoS requirements;

5) To develop a prototype demo implementation for real-time operation and evaluate this prototype with relevant case studies.

\section{Research Methodology}

The proposed approach is broken down into two research steps. The first step is to create a distributed dynamic spectrum access (DSA) mechanism as well as a low-overhead multi-hop routing protocol by which any two far apart D2D users can opportunistically access the spectrum actively in use by cellular users. The second step will develop an opportunistic scheduling based wireless multicast scheme, by which a multicasting transmitter (a BS or a UE) can efficiently transmitting a common set of data to multiple users over fading channels. Besides, a D2D retransmission based multicast scheme is also investigated considering one or very few receiver UEs may be with very poor channel conditions.

\subsection{Development of DSA Mechanism and Routing Protocol for Multi-hop D2D Communications}

The purpose of the study is twofold. First, we will investigate the distributed power control strategies for D2D transmitters which allow D2D links to simultaneously operate in the same frequency band as licensed cellular links. The second is to create an effective route discovery protocol for multi-hop D2D communications, which can reduce the routing overhead, optimize the system performance (e.g., link outage probability) and save energy for D2D transmitters.

On how to set feasible transmit powers for D2D transmitters in a distributed fashion without BS assistance, and on how to achieve a reliable level of performance for D2D users while can also keep the interference to the co-channel cellular users within the allowed level, the working approach will build on our earlier work in [15], which have investigated some of the basic properties of dual-hop relaying based D2D communications. However, D2D users are without any assistance of BSs in emergency environments, due to network congestion or damage of BSs. The non-orthogonally channel share between D2D users and cellular users may cause severe signal-to-noise-and-interference-ratio (SINR) decrease for both the two type of users. To assist D2D transmitters in opportunistically accessing the spectrum in a distributed ad hoc fashion, we will resort to the interference temperature method in the context of cognitive radio [16], and we will propose a novel power control strategy for D2D transmitters. The power control should be able to keep the SINR that the licensed cellular users receive within the allowed level, and, at the same time, the unlicensed D2D users can still achieve a reliable level of performance (e.g., link outage probability).

Base on the proposed DSA mechanism, we will further investigate the route discovery algorithm as well as the network information to develop the routing protocol for multi-hop D2D communications. In PSNs, 
two far apart D2D users will attempt to discover either a single-hop or multi-hop route connecting each other. For that purpose, first, appropriate ad hoc routing protocol such as Dynamic Source Routing (DSR) protocol, Ad hoc On-demand Distance Vector (AODV) protocol or Destination Sequenced Distance Vector (DSDV) protocol should be carefully selected and improved. Then, network information related to the transmit power and the measured interference power of multi-hop D2D relays should be designed. The network information is to be added to the discovery packet of the selected routing protocol in addition to relays' address. It is expected to decrease the probability of failure in finding a route while significantly reducing the number of transmissions necessary to discover a route. With the framework in place to find routes between D2D users, we will further optimize the energy consumption and the outage probability for a multi-hop D2D link.

\subsection{Development of Optimized Opportunistic Multicast Scheduling Scheme for Emergency Group Communications}

The purpose of this research step is also twofold. First, we will investigate the opportunistic multicast scheduling scheme to satisfy the QoS requirement (e.g., minimizing the system delay) of emergency group communications. A common case in PSNs where a UE instead of a BS acts as the multicast transmitter is emphasized. The second mission is to utilize D2D retransmission to further improve wireless multicast service when one or very few receiver UEs are with very poor channel conditions. The optimal number of cooperative relay UEs within a multicast group will be selected to perform dual-hop cooperative relaying, and, hence, to enhance multicast performance.

To implement the first task, we will combine advanced physical layer and MAC layer techniques and employ the cross-layer design theory to design and optimize QoS-oriented multicast scheduling scheme for emergency group communications. In PSNs, it is usually a UE instead of a BS which acts as the multicast transmitter. To reduce the network burden of UE transmitters, we adopt the fountain coding method in the physical layer. Fountain codes allow the distributed multicast receiver UEs to recover the full original content of a message once a minimum set of encoded symbols is received, regardless of the specific received sequence of encoded symbols. If the multicast is initiated by a UE, it does not need to keep track of the data that each receiver UE has received throughout the content delivery process. This obviously reduces the huge network burden for UE transmitters. To achieve this task, the cross-layer design method should be employed in the following algorithm development and optimization.

In literatures, there are two multicast scheduling schemes, i.e., the opportunistic unicast scheduling scheme and the broadcasting scheme. The former exploits multiuser diversity by limiting the multicast transmitter to transmit to the receiver UE with the best instantaneous channel in each time. In contrast, the latter exploits the broadcast gain of the wireless channel by transmitting to all receiver UEs simultaneously. However, the rate is constrained by the UE with the poorest channel. To better balance the tradeoff between the multiuser diversity and the broadcast gain, we will investigate a novel opportunistic multicast scheduling scheme in the proposed approach. Assuming that each user experiences independent fading over different time slots, the proposed scheme will select a different subgroup of users for transmission in each time slot at a rate supported by the worst receiver in the selected subgroup. The objective is to minimize the system delay of emergency multicast services.

Next, to implement the second task, we propose a D2D retransmission based multicast scheme to enhance the throughput of emergency group communications, and we will also analyze the resource utilization efficiency of the scheme with different numbers of D2D retransmitters/relays under different D2D channel conditions. This stage of work will build on our earlier work in [17], which has demonstrated that cooperative relaying can further improve multicast throughput. Although we have investigated the opportunistic multicast scheduling scheme for emergency group communications, the achievable multicast 
throughput may significantly degrade when a UE instead of a BS being the multicast transmitter as the transmission power of a UE is extremely limited. By employing additional D2D retransmissions, we will effectively mitigate this communication bottleneck. A receiver UE in good channel condition is allowed to retransmit its correctly received multicast data to those receiver UEs experiencing relatively poor channel conditions via D2D links, such that the multicast transmitter UE can transmit at a high data rate at low power. Correspondingly, the following two issues are to be investigated: (1) there might be more than one receiver UE that can decode the data correctly. To achieve maximal resource utilization, which of those successful UEs should be designated as the D2D retransmitters and how many? (2) due to differences in fading and path loss, D2D channel conditions may widely vary from one link to another, resulting in very different channel capacities among different D2D links. The D2D retransmission route should be carefully selected to detour those D2D links with poor link quality.

\section{Conclusion}

Since the proposed approach opens many novel research directions towards future LTE-based PSNs, we will in sequel combine the advantages of the information theory, rateless coding theory [18], cross-layer design method [19], convex optimization theory [20] and queuing theory [21], and explore their novel advances in the radio engineering of LTE-based PSNs. The outcomes of this paper can potentially benefit various academics in UK universities and worldwide. The immediate beneficiaries are researchers working in LTE-based emergency communication area. For example, Ryan Seick, Motorola Solutions' product manager and lead applications manager for public-safety devices, can utilize our intelligent radio technologies into his LTE-based PSN platform designs. The proposed approach can also input/contribute to the U.S. next-generation 911 (NG-911) system [22], which is overseen by the First Responder Network Authority (FirstNet) with the purpose of establishing, operating, and maintaining an interoperable broadband PSN.

\section{Acknowledgment}

This work was supported by the National Nature Science Foundation of China (Grant No. 61471361 and 61572505).

\section{References}

[1] Misra, P., Kanhere, S., Ostry, D., \& Sanjay, J. (2010). Safety assurance and rescue communication systems in high-stress environments: A mining case study. IEEE Communications Magazine, 48(4), 66-73.

[2] Doumi, T., Dolan, M. F., Tatesh, S., Casati, A., Tsirtsis, G., \& Anchan, K., et al. (2013) LTE for public safety networks. IEEE Communications Magazine, 51(2), 106-112.

[3] Motorolasolutions. (September 2011). Project 25 Interoperable Communications for Public Safety Agencies (White Paper).

[4] Federal Communications Commission. (September 2012). Report and order-Amendment of Part 90 of the Commission's Rules to permit Terrestrial Trunked Radio (TETRA) Technology.

[5] ETSI EN 300 392-1. (2006). Terrestrial Trunked Radio (TETRA); Voice Plus Data (V+D); Part 1: General Network Design (White Paper).

[6] Doumi, T. L. (2006). Spectrum considerations for public safety in the United States. IEEE Communications Magazine, 44(1), 30-37.

[7] Mayer-Schonberger, V. (2002) Emergency communications: The quest for interoperability in the United States and Europe. International Journal of Communication Law and Policy, 2-9. 
[8] Ljubljana, S. (March 2012). 3GPP activities on emergency services V0.0.1. Proceedings of 3GPP TSG Meeting \#56, MCC (pp. 1-43).

[9] Yaacoub, E., \& Kubbar, O. (2012) Energy-efficient device-to-device communications in LTE public safety networks. Proceedings of IEEE Globecom Workshops (GC Wkshps) (pp. 391-395). Anaheim, CA.

[10] Salkintzis, A. K. (2006) Evolving public safety communication systems by integrating WLAN and TETRA networks. IEEE Communications Magazine, 44(1), 38-46.

[11] Doppler, K., Rinne, M., Wijting, C., Ribeiro, C. B., \& Hugl, K. (2009) Device-to-device communication as an underlay to LTE-advanced networks. IEEE Communications Magazine, 47(12), 42-49.

[12] Lin, X., Andrews, J., Ghosh, A., \& Ratasuk, R. (2014) An overview of 3GPP device-to-device proximity services. IEEE Communications Magazine, 52(4), 40-48.

[13] Nishiyama, H., Ito, M., \& Kato, N. (2014). Relay-by-smartphone: Realizing multihop device-to-device communications. IEEE Communications Magazine, 52(4), 56-65.

[14] UK seeks to replace TETRA with LTE as early as 2016. From http://urgentcomm.com/tetra/uk-seeks-replace-tetra-lte-early-2016

[15] Zhang, G., Yang, K., \& Liu, P. (2013). Power allocation for full-duplex relaying based device-to-device (D2D) communication underlaying cellular networks. IEEE Transactions on Vehicular Technology.

[16] Yang, C. G., Li, J. D., \& Tian, Z. (2010). Optimal power control for cognitive radio networks under coupled interference constraints: a cooperative game-theoretic perspective. IEEE Transactions on Vehicular Technology, 59(4), 1696-1706.

[17] Zhang, G., Yang, K., Hu, Q., Liu, P., \& Ding, E. (2012). Bargaining game theoretic framework for stimulating cooperation in wireless cooperative multicast networks. IEEE Communication Letters, 16(2), 208-211.

[18] Erez, U., Trott, M. D., \& Wornell, G. W. (2012) Rateless coding for Gaussian channels. IEEE Transactions on Information Theory, 58(2), 530-547.

[19] Srivastava, V., \& Motani, M. (2005) Cross-layer design: a survey and the road ahead. IEEE Communications Magazine, 43(12), 112-119.

[20] Boyd, S., \& Vandenberghe, L. (2009). Convex Optimization. Cambridge University Press.

[21] Allen, A. O. (1990). Probability, Statistics, and Queueing Theory: With Computer Science Applications. Gulf Professional Publishing.

[22] Donny, J. (2013). From http://www.nena.org/?NG911_Project

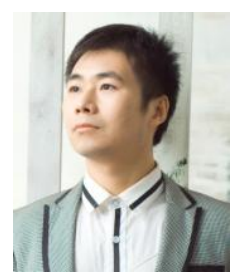

G. Zhang received his Ph.D. from the School of Communication Engineering of Xidian University, China, his M.Sc. from the School of Computer Science of South China Normal University, China, and his B.Sc. from the Department of Computer Science of Jiangsu (original Xuzhou) Normal University, China. He is currently an Associated Professor in the School of Computer Science and Technology of China University of Mining and Technology (CUMT). His main research interests include micro and macro economics, game theory, wireless sensor networks, and cognitive radio networks etc. He has published more than 50 journal papers. He is a member of IEEE.

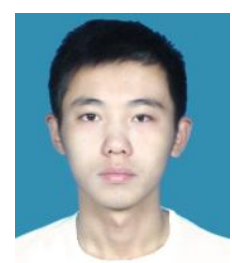

A. Li received his B.Sc. from the School of Electrical Engineering of Anhui Polytechnic University. Now, he is pursuing his M.Sc. in the School of Information and Electrical Engineering of CUMT under the direction of Dr. G. Zhang. His research interests include wireless sensor networks and cognitive radio networks. 
Y. Zhou currently is an Associate Professor at the School of Computer Science and Technology of CUMT. He got the B.Sc., M.Sc., and Ph.D. degrees all from the Department of Electrical Engineering of CUMT. His research interests include machine learning, nonlinear dimensionality reduction and cognitive sciencey.

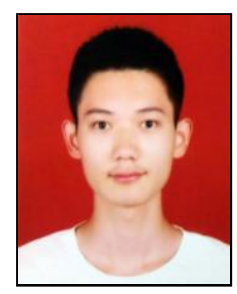

Y. Du is currently an undergraduate in the School of Information and Electrical Engineering of CUMT, and majors in information and communication technologies (ICTs). He is also a member of Dr. G. Zhang's workgroup on D2D communications. 